\title{
ARISTOTE TOPOLOGUE
}

\author{
René Thoм
}

Résumé : René Thom scrute les propriétés qu'on peut assigner à tout lieu, et désigne la question de ses bords, celle des extrémités. Il s'interroge sur la théorie aristotélicienne des lieux que la transposition topologique éclaire. Des réflexions sur le lieu propre à un animal et des impressions de voyage de Marcel Proust étayent la construction.

Mors-CLés : lieux, bords, amers, topologie, formule de Stokes, Aristote, Marcel Proust, Raymond Roussel.

ABSTRACT : René Thom scrutinizes the properties which can be assigned to each location, and designates the question of its peripheries, that of its extremities. He questions the Aristotelian theory of locations which a topological transposition clarifies. Reflexions on the location proper to an animal and impressions of Marcel Proust's voyage back up this construction.

KEYWORDs : locations, peripheries, sea-marks, topology, Stokes' formula, Aristotle, Marcel Proust, Raymond Roussel.

Revue de synthèse : $4^{e}$ S. $n^{0} 1$, janv.-mars 1999, p. 39-47. 
ZuSAMMENFASSUNG: René Thom untersucht, welche Eigenschaften jedem Ort zugeschrieben werden können; es geht ferner um die Begrenzung und die vom Zentrum entfernten Bereiche. Er fragt nach der Aristotelischen Theorie der Örter und deren Erklärung durch topologische Übertragung. Die gedankliche Konstruktion wird unterstützt durch Überlegungen über den Ort bei den Tieren, und über Reiseeindrücke von Marcel Proust.

STICHWÖRTER : Örter, Ränder, Landzeichen, Topologie, Stokesche Formel, Aristoteles, Marcel Proust, Raymond Roussel.

RIASSUNTO: René Thom scruta le proprieta che si possono assignare ad ogni luogo, e indica la questione dei suoi bordi, quella delle sue estremità. Si interroga sulla teoria aristotelica dei luoghi che la trasposizione topologica chiarisce. Alcune riflessioni sul luogo proprio ad un animale ed alcune impressioni del viaggio di Marcel Proust illustrano la costruzione.

Parole chiave : inoghi, bordi, amari, topologia, formula di Stokes, Aristotele, Marcel Proust, Raymond Roussel.

Ancien élève de l'École normale supérieure, médaille Fields en 1958, le mathématicien René Тном est actuellement professeur à l'Institut des hautes études scientifiques de Bures-surYvette.

Adresse : Institut des hautes études scientifiques, 35 route de Chartres, F-91440 Bures-surYvette. 
Aristote n'a pas imaginé le mot topos. Il l'a trouvé, existant en grec, avec son sens de lieu, alias localité. Citation du Dictionnaire de Bailly: Elládos topoi (chez Eschyle). Il est en conséquence légitime d'examiner la séquence : grec $\rightarrow$ topos, latin $\rightarrow$ locus, français $\rightarrow$ lieu, afin, si possible, de préciser le sens de l'évolution de cette racine.

Si l'on examine les emplois actuels du mot lieu en français, on observera qu'un lieu demande toujours un habitant qui en fait sa résidence : il est pratiquement impossible, en français actuel, d'employer le mot lieu avec un génitif inanimé. Exemples : le lieu d'un rocher, le lieu d'une tour. Dans ses emplois abstraits, philosophiques, le mot lieu, en français, a toujours une forte connotation existentielle : il y a « lieu de " pour agir, « il n'y a pas lieu de » négativement. De là, l'hypothèse que le mot topos implique virtuellement un être humain ou un animal qui séjourne (normalement) en ce lieu; nous l'appellerons ici le maître (ou le chef) du lieu.

De l'humain, on généralisera à l'animal : tout animal se forge un territoire, lieu de ses activités. Il faut concevoir que tout être vivant est ainsi le centre d'un domaine tridimensionnel - faut-il dire son topos? -, où il pratique ses activités de reproduction, de chasse, etc. Un tel domaine doit être vu comme un topos doté d'une position centrale, émettant des ramifications nourricières, et éventuellement familiales. De ce fait, tout topos vit dans la cervelle de son maître occupant. Les incursions externes de l'occupant seront limitées par des objets fixes « extrêmes », à ne pas dépasser, les eschata.

On peut partir de l'hypothèse (simpliste) qu'Aristote, s'imaginant un être vivant, le dotera d'un territoire. Initialement, il est seulement possible d'attribuer à l'être vivant une certaine liberté de déplacement à l'intérieur d'un domaine (ouvert connexe) de l'espace euclidien usuel. Mais ce domaine aura, dans la pratique, des bornes que l'individu préférera ne pas franchir. De là la notion - si importante chez Aristote - de bornes extrêmes, de limites : les eschata.

L'exigence pour un lieu d'avoir des eschata ne paraît ainsi qu'une forme locale, affaiblie, de l'exigence de fermeture qui caractérise pour Aristote l'ousia: Tode ti kekhôrismenon, ce qui est là, séparé. Il en ira de même pour l'emploi «absolu»du mot, «Des Lieux les absolus lieux » selon Mallarmé, où, bien au contraire, la présence d'un individu agissant, limité d'eschata solides proches ou lointains, apparaît comme essentielle. Dorénavant, cet individu agissant sera désigné comme le maître du lieu.

Il nous faut maintenant expliciter la structure des eschata dans un lieu, laquelle présente a priori une surprenante duplication : la double couronne des eschata. 
Une sorte d'enveloppe convexe (dans la terminologie des géomètres d'aujourd'hui) constitue la zone des extrémités, eschata, que l'occupant se garde en général de franchir. Il s'agit là de la notion de «bord » ou de « frontière ». Il sera expliqué plus bas comment les eschata peuvent servir le chef du domaine à se repérer dans son territoire.

Dès le Parménide apparaît le premier vocable de la topologie grecque, le sunekhes, usuellement traduit par continu. Cet usage est malheureux, car c'est plutôt la connexité (par arcs) du domaine qui est évoquée par sunekhes. Les hardis navigateurs de la mer Égée pouvaient vérifier qu'une île est connexe, en en faisant le tour...

Dans mon premier article consacré à la théorie des lieux chez Aristote, et accepté dans la Revue thomiste des aristotéliciens de Toulouse, je n'avais pas résisté au plaisir d'identifier une formule d'Aristote à une célèbre formule de topologie - la formule de Stokes (en homologie) -, à savoir $\mathrm{d} O \mathrm{~d}=0$. Pierre Aubenque m'a alors reproché $\mathrm{d}$ ' aller plus vite que la musique ». Il est de fait qu'à l'époque classique de Platon et d'Aristote, les géomètres semblent avoir manifesté une certaine répugnance à faire coïncider deux de leurs points, une notion pour laquelle ils n'avaient apparemment pas de mot adéquat. Il n'y avait pas alors de verbe pour signifier la coïncidence de deux points. Plus tard, à l'époque d'Euclide, on n'a pas hésité à déplacer des figures entières considérées comme des solides, par exemple en faisant glisser une équerre sur une règle (verbe ephharmôzein). Une lecture rapide de la formule (Physique 211b 12): En tautô gar ta eschata tou periekhontos kai tou periekhomenou, m'avait fait croire (« le bord du corps enveloppé et le bord du corps enveloppant sont ensemble ») qu'on pouvait identifier géométriquement ces deux bords. Je dois à Pierre Aubenque - qui s'était alors intéressé à mon travail - de m'avoir convaincu qu'il n'en était rien. Les deux bords sont «proches », mais ne coïncident pas! Bien mieux, c'est leur distinction même qui va produire la structure «d'identité binaire interne» (en tautô) que signifie en fait l'adverbe « ensemble».

Cela demande une discussion préliminaire sur la « coïncidence » de deux points.

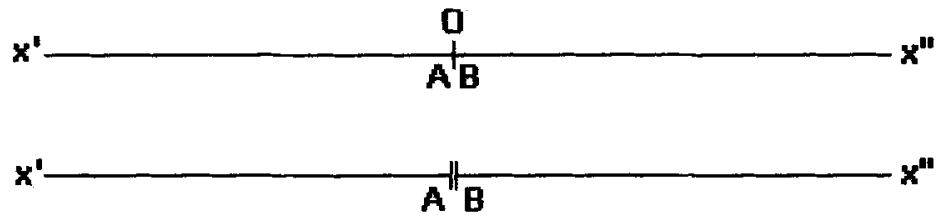

Fic. 1. - Tracée au tableau pendant l'exposé. 
Il faut rappeler ici l'expérience mentale qui soutient l'affirmation l'entéléchie sépare, Métaphysique $\mathrm{Z}, 13,1039 \mathrm{a}, 6-7$ : on marque l'origine $\mathrm{O}$ sur l'axe x'Ox", Aristote introduit deux points bouts A et B pour les demi-axes x'A et Bx" que l'entéléchie de la coupure en $\mathrm{O}$ a fait naître (voir fig. 1, p. 42). Ces points bouts sont distincts et cependant ensemble (hama). Le problème de points qui seraient à la fois distincts et ensemble se pose donc en termes classiques, mais, il est vrai, la nature spéciale des points bouts (A) et (B), fabriqués comme points limites à l'infini (par dérogation à l'injonction aristotélicienne : Mè eis Apeiron ienai!), explique le paradoxe !

Le même paradoxe a lieu pour l'identification présumée de la maxime $211 \mathrm{~b} 12$ : en tautô gar ta eschata tou periekhontos kai tou periekhomenou (le bord $\mathrm{du}$ corps enveloppé et le bord du corps enveloppant sont « ensemble »). Ici encore, on a, avec en tautô, l'existence d'une dualité plaquée sur l'intervalle que j'avais cru devoir réunir. En fait, seule une technique d'orientation, une motivation inspirée des amers maritimes me semble concevable. Ce qui est en jeu ici, c'est l'usage des eschata dans le repérage de son domaine que fait l'usager maître du lieu.

$\mathrm{Au}$ fond, la théorie aristotélicienne des lieux souffre d'une ambiguïté essentielle : s'agit-il d'une théorie d'inspiration strictement géométrique au sens moderne du terme ou, au contraire, d'une théorie de type éthologique liée à l'usage de l'espace par l'être vivant? Dans un article antérieur, j'avais pris un point de vue biologique que je croyais plus pertinent à la philosophie propre d'Aristote. Il en est résulté que la théorie des lieux apparaît souvent comme le locus horribilis de l'aristotélisme, et mise à part la thèse latine de Henri Bergson, Quid Aristoteles de loco senserit (1889), elle n'a guère eu d'écho. Peut-on voir présentement dans cette théorie autre chose qu'un monolithe désuet de l'aristotélisme primitif?

En fait, selon la conception ici proposée, la théorie des lieux serait liée à un problème central de l'éthologie actuelle : comment un animal (ou un humain) se repère-t-il au sein de son territoire?

Le seul principe directeur, nous espérons le montrer dans ce qui suit, expliquera cette construction intellectuelle, apparemment bizarre, qu'est la theory of places.

Il faut trouver la raison d'être des eschata. Les oiseaux ont la barrière du nid, laquelle joue un grand rôle dans l'éducation de leurs petits. Un latiniste pourrait certes objecter à ma thèse sur le lieu nécessairement habité l'exemple du titre Locus Solus (1914) de Raymond Roussel. Certes, Locus Solus signifie lieu inhabité. Mais l'usage est surréaliste : il s'agit d'un grand domaine en banlieue parisienne où toutes sortes d'activités techniques et historiques vont prendre naissance et proliférer dans l'espacetemps.

Un éthologue moderne pourra se demander pourquoi des eschata? La théorie ci-dessous va tenter d'expliquer la nécessité des eschata. Dans ce 
but, on peut introduire un document "littéraire " assez classique, un texte de Marcel Proust, extrait d'À la recherche du temps perdu. Du côté de chez Swann (Paris, Gallimard, «Bibliothèque de la Pléiade », 1987, p. 181182) :

«Seuls, s'élevant du niveau de la plaine et comme perdus en rase campagne, montaient vers le ciel les deux clochers de Martinville. Bientôt nous en vîmes trois; venant se placer en face d'eux par une volte hardie, un clocher retardataire, celui de Vieuxvicq, les avait rejoints. Les minutes passaient, nous allions vite et pourtant les trois clochers étaient toujours au loin devant nous, comme trois oiseaux posés sur la plaine, immobiles et qu'on distingue au soleil. Puis le clocher de Vieuxvicq s'écarta, prit ses distances, et les clochers de Martinville restèrent seuls, éclairés par la lumière du couchant que même à cette distance, sur leurs pentes, je voyais jouer et sourire. Nous avions été si longs à nous rapprocher d'eux, que je pensais au temps qu'il faudrait encore pour les atteindre quand, tout d'un coup, la voiture ayant tourné, elle nous déposa à leurs pieds; et ils s'étaient jetés si rudement au devant d'elle, qu'on n'eut que le temps d'arrêter pour ne pas se heurter au porche. Nous poursuivîmes notre route; nous avions déjà quitté Martinville depuis un peu de temps et le village après nous avoir accompagnés quelques secondes avait disparu, que restés seuls à l'horizon à nous regarder fuir, ses clochers et celui de Vieuxvicq agitaient encore en signe d'adieu leurs cimes ensoleillées. Parfois l'un s'effaçait pour que les deux autres puissent nous apercevoir un instant encore; mais la route changea de direction, ils virèrent dans la lumière comme trois pivots $d^{\prime}$ or et disparurent à mes yeux. »

Ce passage est apparu à Proust comme sa première contribution d'auteur à son œuvre. Ici, nous l'introduisons comme pouvant s'expliquer par l'exemple d'une technique d'orientation marine : les amers.

La technique des amers est une technique de repérage en usage chez les navigateurs. Soit une vaste baie limitée vers le haut par l'Océan, et trois points océaniques I, J, K, dotés chacun de phares puissants tous identifiables. Il importe alors d'étudier le système des droites, dites « amers », engendré par un tel trio de points. Il y en a trois: IJ, JK, IK. Sur la côte supposée rectiligne, on distinguera chaque type de point $P$ par l'ordre (gauche-droite) selon lequel on voit les divers rayons PI, PJ, PK. Finalement, la position d'un point $M$ sur la rive du continent par rapport aux trois phares IJK est définie par la succession des sources reçues par un observateur de la rive, qui balaie du regard, de gauche à droite, l'horizon marin (voir fig. 2, p. 45). En se déplaçant sur la rive, disons d'est en ouest, l'observateur verra coïncider deux des phares lorsqu'il se trouve précisément sur une droite amer. Bien mieux, si la visibilité sur la terre ferme le permet, il pourra numéroter les types d'ordre selon lequel il aperçoit les trois sources, les “ permutations » de sources; cela lui permet d'arriver à une classification qualitative des ensembles perçus, en algèbre : une « per- 
mutation » des sources. Certaines des zones ainsi définies sur le continent peuvent être très étroites et, par conséquent, donner des repères topographiques qualitativement bien précis.

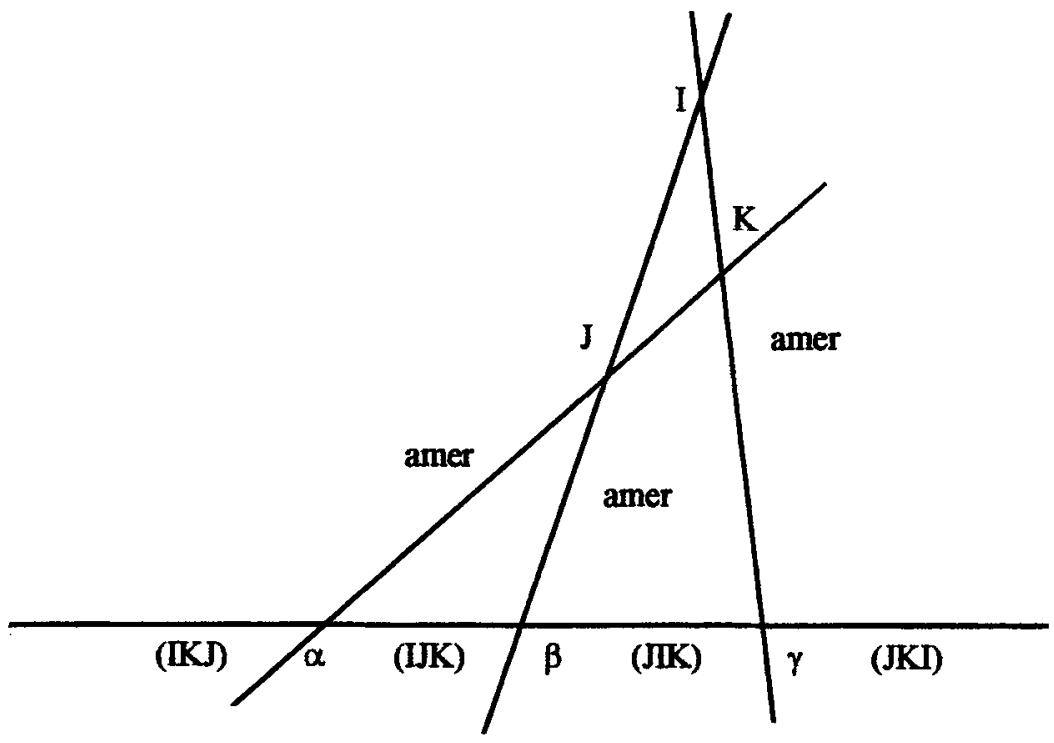

Fig. 2. - Amers.

On conçoit qu'avec un tel système, le "maître " d'un territoire puisse, en procédant à une analyse de ses eschata ordonnés selon la vue, aboutir à une évaluation qualitative correcte de la zone où il se trouve. Évaluation qualitative, certes, mais qui suffit pratiquement en presque tous les cas.

Il n'est pas dans mon propos ici d'étayer cet argument en faveur d'une interprétation qualitative des phénomènes observés par Proust dans sa promenade du soir à Martinville. Il y faudrait une étude locale raffinée qui a peut-être déjà été faite. On retiendra de notre analyse des amers qu'en un certain sens, il existe une sorte de surjection des eschata extérieurs sur les eschata intérieurs, laquelle détermine les places usuelles du chef au cours de ses déplacements dans son territoire. Qu'on rigidifie cette surjection en une fibration, alors la structure entauta de la différence eschata externes $\rightarrow$ sur eschata intérieurs pourrait prendre la forme requise pour mon interprétation : on aurait alors un collier régulier pour cette différence globali- 
sable - ce qui entraînerait la formule de Stokes, homologique : le bord $d u$ bord est vide... Nous verrons que tel est bien le cas.

$S$ 'il est aisé de comprendre en quoi les amers peuvent donner au chef des moyens pour localiser sa propre position au sein d'un lieu, on comprendra moins bien le rôle des eschata intérieurs, à savoir la limite minimale du « corps enveloppant », telle qu'elle est prescrite en Physique 211b 11.

Sous cette forme, il est difficile de comprendre la nécessité de ces deux types d'eschata. Si les eschata externes peuvent servir à construire des amers, grâce auxquels on se localisera, tant mieux! Par contre, on voit difficilement à quoi peut servir cette distinction concernant l'usage du « premier » organisme enveloppé.

Il faut certainement faire ici une hypothèse hardie : quel peut être le premier organe enveloppé ? Il faut s'imaginer ici le maître du lieu se déplaçant selon quelque sentier ébauché en son lieu. En ce cas, le premier organe enveloppé, c'est son œil sous sa paupière. Il faut s'imaginer le maître visant une direction externe, un eschaton lointain. Alors le rayon visuel du maître va connecter l'œil du maître et le repère du bord lointain : Aristote sait que le rayon lumineux est comme une canne qui vient buter sur l'objet, répercutant le choc de l'eschaton lointain au nerf sensible sous la pupille - la korè kosmou - l'eschaton premier sous la paupière; le rayon lumineux variable forme ainsi un collier reliant les deux bords - eschata (réversibilité que connaît bien la physique moderne, mais beaucoup plus surprenante chez Aristote). Une fibration en rayons lumineux va ainsi connecter les deux bords : externe et interne. Grâce à quoi on pourra - en topologie moderne - les identifier, comme l'exigeait la version homologique de la formule de Stokes (le bord du bord est vide).

Cette formule exprime essentiellement le caractère clos de l'être vivant. Car s'il y a un bord, il y a perte de sang, avec menace pour la vie ${ }^{1}$. D'où le rôle de détecteur d'ontologie qu'est l'opérateur bord $\left(\mathrm{d}^{2}=0\right)$ de l'algèbre homologique et sa profonde interprétation biologique...

Cette algébrisation de la théorie pourra sembler arbitraire. Elle témoigne du fait que ce type d'étude n'a pas été pris en considération par les éthologues (pas assez « scientifique »?). Pourtant, il y a eu des études considérables sur la barrière du nid chez les oiseaux. Et, par ailleurs, les phénomènes observés lors du déplacement « en aveugle » d'une ruche témoigne d'une démarche des abeilles butineuses qui s'appuie sur des indices externes de la végétation. Il ne fait guère de doute que ce problème du repérage de ses lieux par l'animal (et par l'homme) attend encore des recherches compréhensives, mais il faudrait pour cela donner à l'éthologie

1. On pense à cette réflexion de Woody Allen, entendue dans son film Bananas : s'étant coupé la main en jouant avec un couteau, il y voit perler quelques gouttes de sang. Il pousse alors cette expression craintive : "Ehh, it should be inside...!" 
animale un statut plus conceptuel qui la fasse sortir d'une expérimentation trop immédiate.

Quant à moi, j'aurais voulu faire sortir la théorie des lieux d'Aristote de son statut de locus horribilis de l'œuvre du Philosophe. Je me borne ici à espérer que, parmi mes lecteurs, certains penseront qu'il y a là des directions qui mériteraient d'être explorées...

On peut se demander comment Aristote concevait la continuité du mouvement «selon le lieu», la phora. Quand le mobile quittait un lieu pour entrer dans un autre, s'agissait-il d'une transformation continue, ou, au contraire, marquée par une discontinuité? Notre point de vue qui rend le maître du lieu assujetti aux eschata du lieu plaide pour une discontinuité essentielle dans le passage d'un lieu à un autre. Car quittant un lieu (L1) pour entrer dans un lieu (L2), le marcheur doit d'abord remplacer les eschata (extérieurs) de (L1) par ceux de (L2), ce qui oblige à une discontinuité « catastrophique ", si modeste soit-elle par ses effets physiologiques.

René Тном (mai 1996). 